\title{
Use and Perception of the DCRB Core Standard
}

\section{Winslow Lundy}

In January 1999, the Program for Cooperative Cataloging approved the core bibliographic standard for rare books, called the DCRB Core standard. Like the other core standards, the DCRB Core provides the framework within which catalogers can create bibliographic records that are less than full, but are as reliable as full-level records in description and authorized headings. In the three years since its approval, there is little evidence that the standard has been widely used. This study reports the results of a survey sent to forty-three participants who indicated in a preliminary query that they do use the DCRB Core or that they have made the decision not to use it. In the thirty-seven surveys that were returned, only about 16\% of the respondents said they have used the standard to create bibliographic records for their rare books. The libraries that do not use the core standard find it inferior or lacking in a number of ways. Several of those libraries, however, are planning to use the standard in the future or are seriously planning to investigate using it. Such intent may indicate that the time is approaching when more libraries will find reasons to implement the standard. One impetus may come from the findings of a recent survey of the special collections departments of member libraries of the Association of Research Libraries that emphasize the size of the backlogs in those departments. If faster accessibility to specific portions of the backlogs would benefit users more than having fulllevel cataloging, application of the DCRB Core standard could facilitate reducing those backlogs.

B ibliographic control of rare book collections has always been a time-con-

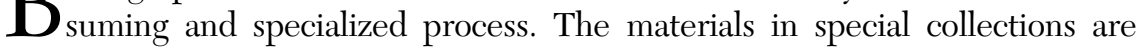
there for particular and compelling reasons. Catalogers of such collections have traditionally identified those special attributes in catalog records with full-level bibliographic description, extensive notes, and as many access points as are deemed appropriate by both catalogers and curators. The bibliographic standards for cataloging rare books, with the principles of Anglo-American Cataloguing Rules, 2d edition (AACR2) underlying them, have evolved over a number of years, first in 1981 in Bibliographic Description of Rare Books and most recently in Descriptive Cataloging of Rare Books (DCRB), published in 1991. A new edition, with the title Descriptive Cataloging of Rare Materials, is now in preparation by the Association of College and Research Libraries, Rare Books and Manuscripts Section (RBMS), Bibliographic Standards Committee. The new edition will include rules for the various formats of materials. The documentation for books will be known as $\operatorname{DCRM}(B)$. While the current standard of DCRB is written primarily for books printed before 1801, it can be used for post-1800 imprints as well.

The Program for Cooperative Cataloging (PCC) has defined and approved core standards for many of the bibliographic formats, beginning with the stan-
M. Winslow Lundy (windy.lundy@colorado.edu) is Assistant Professor and Monographic Cataloger for the University of Colorado at Boulder Libraries.

Manuscript received September 12, 2002: manuscript accepted November 14,2002 
dard for books in the mid-1990s. All the core standards, written for use by the participants in the Bibliographic Records Cooperative (BIBCO) program, are intended to encourage "faster, better, cheaper" cataloging (PCC 1999b). They ensure the same reliability in description, authorized headings, and call numbers as full-level records, but they do not require as many notes or subject headings. In January 1999, the PCC approved the Core Standard for Rare Books. Called DCRB Core because it is based on the full standard of DCRB, the standard is intended for use in cataloging books with imprint dates between 1500 and 1800. The text of the standard was written and proposed by the Task Group for Developing a Standard for Core Treatment of Rare Books, a group that was charged with the creation of the core standard by the PCC Standards Committee and that included several members of the RBMS Bibliographic Standards Committee. The expected use of the DCRB Core standard is for cataloging books that have been chosen more for their need of accessibility than for their full bibliographic details (PCC 1999a).

\section{Definition of the DCRB Core Standard}

Two documents define the core record for rare books: Core Standard for Books (Books Core) and the DCRB Core. The Books Core requires authorized headings, a call number from a standard classification system, full fixed-field data, descriptive fields 245-4XX (title, edition, imprint, physical description, and series), but fewer notes (500, for source of title if not from the title page; 502 , dissertation note; 505, contents note for multipart works with individual titles; and 533, reproduction note), only one or two subject headings, and as many added entries as the cataloger judges to be appropriate (PCC 1996). Significantly, notes for justification of added entries are not required. The frequently occurring bibliographical references note (504) is also not required.

With the requirements of the Books Core as a base, the DCRB Core standard specifies additional or differing elements. Figure 1 presents the DCRB Core elements arranged in MARC tag order (PCC 1999a), with an indication of how or why the elements should be used. The code "dcrb" in field $040 \$ \mathrm{e}$ is mandatory because the bibliographic description is based on the full standard of DCRB. Differing from the Books Core, the DCRB Core does not require a standard call number. Many libraries do classify their rare books, but other libraries use local call numbers. The DCRB Core standard does not require that catalogers who use local call numbers go an extra step and assign a standard call number as well. The title, edition, imprint, physical description, and series areas are all required if appropriate to the item being cataloged, just as they are in the Books Core, but their inclusion follows the descriptive method prescribed by DCRB and may apply the options for shortening the bibliographic record (ACRL RBMS BSC 1998b). The DCRB Core standard specifies two note fields (5XX) in addition to those required by the Books Core. If the cataloger transposes the elements on the title page in the transcription in the title field (245) of the bibliographic record, the transposition must be acknowledged in a 500 field. The citation/references note field (510) provides, in standard form, reference to bibliographic sources. In a fulllevel DCRB record, the 510 field may provide justification for information included in the catalog record. In a DCRB Core record the same reference may lead the user to information that has been omitted from the record, but DCRB Core requires only the five sources listed in DCRB rule 7C14 for post-1500 imprints. The final element of the DCRB Core standard, the index term field (655) that employs genre, form, or physical characteristic terms from standard thesauri, is encouraged but not required.

DCRB Core defines the minimum requirements for the bibliographic record. At the discretion of the cataloger, more note fields (5XX fields) and more access points (subject headings (6XX fields) and added entries (7XX fields)) may be added to a given record. This flexibility means that a core record may contain more than the minimally required fields without attaining the level of a full record.

Examples of a full-level DCRB bibliographic record (figure 2) and its corresponding DCRB Core record (figure 3) illustrate significant differences between the two standards. A DCRB Core record can be recognized by the encoding level code "4" (MARC and RLIN Leader/17 or OCLC fixed field, Elvl) and "dcrb" in the cataloging source field $040 \$ \mathrm{e}$. The examples used here have the appearance of OCLC records,

\begin{tabular}{ll}
\hline 040 \$e dcrb & Cataloging source: Description convention \\
& Mandatory (since the full standard is DCRB) \\
050, 082, 086, 090, etc. & Call number fields \\
& Not required (because many rare book col- \\
& lections use special call numbers) \\
& Title, edition, imprint, physical descrip- \\
& tion, and series fields \\
(following the more inclusive descriptive & method and extent for recording biblio- \\
245-4XX & graphic details and the options prescribed by \\
& DCRB) \\
& Note field \\
& Mandatory (if elements on the title page \\
& have been transposed in the 245 field) \\
& Citation/references note field \\
& Mandatory (if one of the five citations \\
$\mathbf{5 0 0}$ & specifically required by DCRB) \\
& Index term-Genre/form term \\
& Not required but encouraged
\end{tabular}

Figure 1. Elements of the DCRB Core Standard Required in Addition to the Requirements of the Books Core 
and the full-level record has been adapted from an existing OCLC record. Other examples illustrating the differences between the two standards can be found on the Web pages of the RBMS Bibliographic Standards Committee (Fletcher 1999).

The difference in the length of the two records is immediately noticeable. The core record has been created by using the options in the full DCRB standard for shortening the bibliographic description in several areas and by omitting fields not required by the core standard. In the publication area (field 260), the cataloger has followed the option in rule 4C6 in the full standard to shorten the field. Instead of transcribing all six names of the printers of the book, the cataloger has given the name of the first printer and a bracketed statement that there are five other printers. The DCRB Core standard does not require any of the thirteen notes used in the full-level record, including the notes that justify the main (100) and added (700) entries. The thirteen note fields $(5 \mathrm{XX})$ in the full-level record have been reduced to one note in the core-level record, the 510 field (the citation/references note). While the 510 is not one of the five citations required by the full standard (rule $7 \mathrm{C} 14$ ), the cataloger has chosen to include the 510 that gives the reference to the bibliography of Daniel

Defoe because the bibliography contains substantially all of the information in the other notes in the full-level record. Even though this work is a multipart title, the contents note

Figure 2. DCRB Full Record

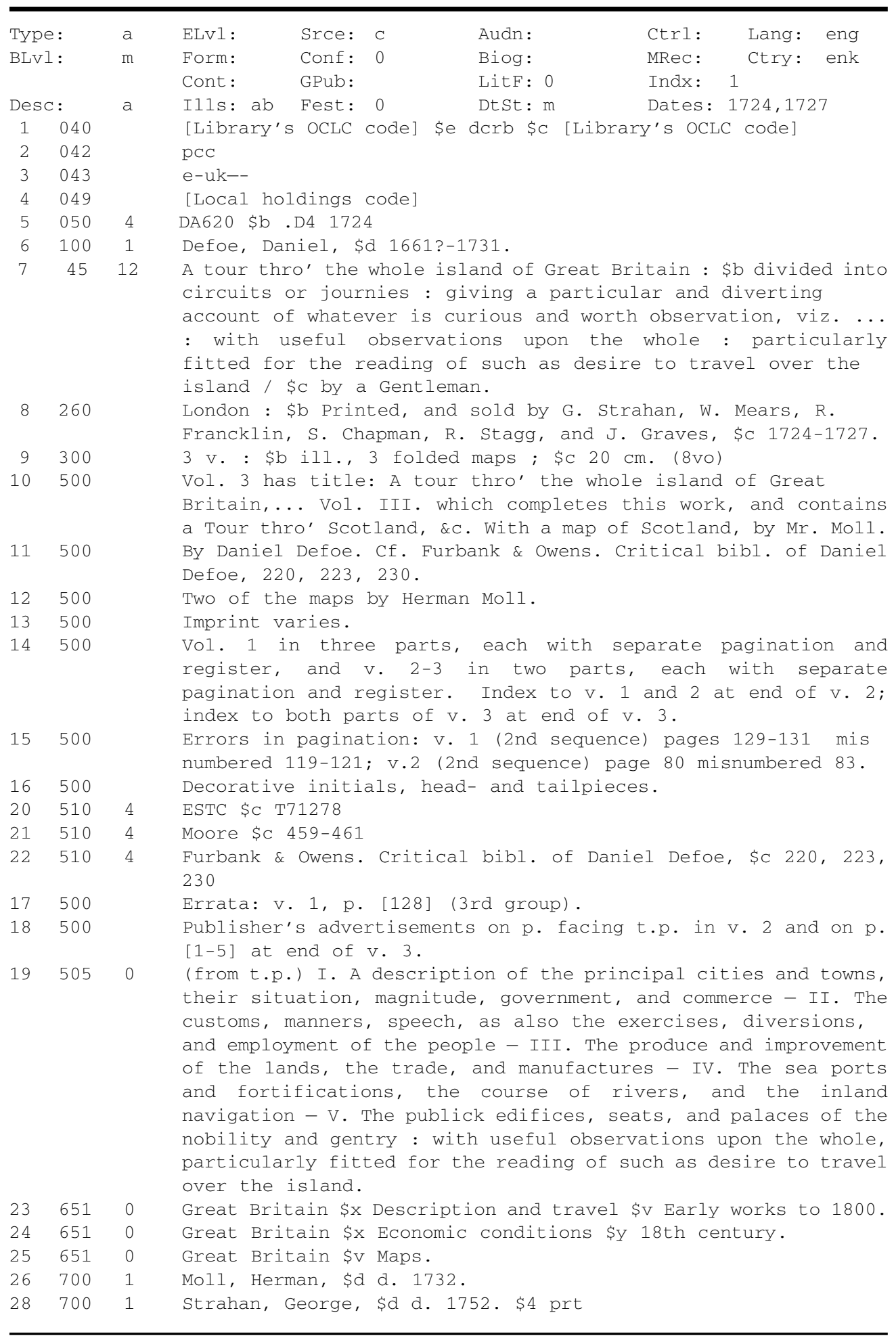

(505) as taken from the title page does not reflect distinct titles of the individual volumes. The contents note, therefore, has been left out of the core record. The DCRB Core record 
contains only one of the subject headings (field 651) and one of the added entries (field 700) used in the full-level record. While the examples are coded as BIBCO records ("pcc" in the 042 field), any library that is not a participating BIBCO member may also create core records. The 042 will be absent from a non-BIBCO record.

Creating a core bibliographic record such as the one in figure 3 would obviously take much less of the cataloger's time. The time savings and therefore the cost savings may be significant factors for some libraries. To implement the standard, however, may alter the past practice of describing a book to the fullest to help users find the edition or issue of a title that they are looking for. The DCRB Core standard was written to give catalogers the option and discretion of using a less-than-full standard when collections have been identified as more important for access than for fuller bibliographic details. The flexibility of adding more to the core record and the dependence on the cataloger's judgment in choosing to add more are two key principles of all the core standards. These two characteristics may make use of the DCRB Core, in particular, an attractive alternative to the time-consuming full-level record.

\section{Literature Review}

Although a number of articles have been written about the Books Core and other core standards, little has been published about the DCRB Core standard. When the standard was being written, discussion and reports at the RBMS Bibliographic Standards Committee meetings were recorded in the minutes of the committee (ACRL RBMS BSC 1998a). The work of the DCRB Task Group was documented in its final report (PCC Task Group 1998). During the time of the task group's work on the standard, discussion and comments were solicited on the Exlibris list. All of those exchanges are available in the archives of the list (Exlibris 1997-1998). After the standard was written and approved, discussion concerning issues of its actual implementation and use was not initiated on Exlibris or Autocat, two lists to which many rare book catalogers subscribe.
Much of the literature treating the core record describes its development within the context of the history of the PCC, its purpose in encouraging "faster, better, cheaper" cataloging that can be relied on for quality of description and authorized headings, and its potential contribution to the success of the national program. There are, however, some studies and reports that investigate particular issues that are relevant to the DCRB Core. Several studies have been done to evaluate the time savings and cost-effectiveness of the core record. Thomas (1996, 102) notes that an unpublished study done at Cornell University by Boissonnas found that core records could be created $25 \%$ faster than full-level records. The UCLA/OCLC Core Record Project (Kelley and Schottlaender 1996), conducted from December 1994 to April 1995 with the same group of catalogers creating core- and full-level records, confirmed the assumption that core-level cataloging is faster than full-level cataloging (by $8.5 \%$ to $17 \%$ ) and further confirmed that fewer subject headings and added entries are used in the core records. Other libraries' subsequent use of those core records in the OCLC database with little additional editing attested to the reliability of the records. Hyslop (1997) discusses an unpublished study of experimental core cataloging done at the Library of Congress in 1996. The experiment underscored the efficiency and productivity of cataloging using the core standard. Cataloging statistics at Colorado State University over several years indicated that the use of the Books Core to catalog government publications primarily in the backlog was successful in making 
a greater number of books accessible more quickly than before the core standard was used (Lange 1998).

Two recent studies explore the sufficiency of the core record. The first study analyzes the access points and notes in the core- and full-level records and finds statistically significant differences in the two levels of records (Czeck, Icenhower, and Kellsey 2000). While the authors indicate the fields that catalogers may want to augment, they acknowledge that catalogers may want to accept basic corelevel requirements unless the need for additional fields outweighs the time savings in the creation of core records. The second study is a pilot study of library users' opinions about the usefulness of the various elements in the core and full records (Letarte and Turvey 2001). Although the study finds the need for further research to confirm the preliminary indications about users' preferences concerning the usefulness of the various access points in the bibliographic records, it does indicate that even though users prefer fulllevel records, they still feel the core record is sufficient for finding the materials they need.

In a study conducted by interview of cataloger and cataloging manager attitudes toward the BIBCO core record, Banush (2001) found that both groups of participants, while generally satisfied with the core record, expressed varying opinions about its problems and benefits. The cataloging managers expressed more satisfaction with the core record than did the catalogers. Banush notes that this attitude of satisfaction with the core record is in contrast to the reduced percentage of core records actually created by the BIBCO participants during 2000 and early 2001. He also reports that there is a distinction between those accepting core records created by other libraries as copy cataloging and those creating original core bibliographic records. Even though libraries readily use other libraries' core contributions to the shared cataloging databases, often without revision, they are more reluctant to create them, preferring instead to create fulllevel records. Cromwell $(1994,423-24)$ reports that even before the Books Core standard was implemented as part of the BIBCO program, Stanford University used a similar standard with varying success. She suggests that the use of the core record cannot achieve the level of cost-effectiveness that is expected without the acceptance and understanding of the catalogers themselves about its purpose and its concomitant emphasis on catalogers' judgment and flexibility.

The published and reported studies of the efficiency and cost-effectiveness of creating core records point to an encouraging potential for the application of all the PCC core standards. The research about access points, users' perceptions, and practitioners' opinions tempers the findings of the efficiency of the core record while producing some statistical and qualitative data that encourages further study.

While the DCRB Core standard bears the approval of the PCC to be used by the libraries participating in the
BIBCO program, it may be applied by any library, whether a BIBCO participant or not. In the three years since the approval of the DCRB Core standard by the PCC, however, there has been little evidence that the standard is being widely used. This study investigates the trends in the use of the DCRB Core by means of a survey designed to investigate catalogers' use and perceptions of the standard.

\section{Method}

To reach catalogers and special collections librarians who would be able to provide information about their experience with the DCRB Core standard, a query was posted to three lists: Autocat, Pcclist, and Exlibris. Although many people subscribe to all three lists, the profile of subscribers is different for each. Autocat is a list of several thousand people worldwide who are interested in cataloging issues; Pcclist is a list whose subscribers are from participating PCC libraries; and the subscribers to Exlibris are those who have an interest in the field of rare books and special collections. In November 2001, the query was posted to the three lists, asking three questions: (1) if anyone has cataloged using the DCRB Core standard; (2) if not, has a decision been made not to use the standard; and (3) if either is the case, would the recipient be willing to participate in a longer survey about the use and perceptions of the DCRB Core. The questions were posed in this way to find catalogers who have given some thought to the use of the core standard, whether or not they actually use it. The intent was not to seek responses from catalogers who do not use the core and have never considered doing so. Within a reasonable amount of time, only 15 libraries had sent responses, and of those, only 4 indicated that they use the DCRB Core standard. Thirteen, however, said they were willing to participate in a survey.

A few respondents to the query to the three lists indicated that they were responding because someone else had forwarded the query to them. Several more focused groups of potential respondents, therefore, were polled by e-mail: the liaisons at the BIBCO libraries, the members of the RBMS Bibliographic Standards Committee for the past several years, and the heads of cataloging, heads of special collections or special collections catalogers at member libraries of the Association of Research Libraries (ARL). Using this second method, 135 queries were sent to the three groups. Sixty-five libraries responded and, of those, 30 indicated that they would participate in a survey. By using these two methods, posting to the lists and soliciting individual libraries, 43 libraries were identified that were willing to participate in the survey. In March 2002, the 43 surveys were distributed to the participants as Word and rich-text-format attachments to e-mail messages. All participants were assured that 
their names and the names of their institutions would be kept confidential. Thirty-seven surveys were returned.

Since most of the solicited libraries are large research institutions, the majority of the returned surveys are from that category of library. The responses to the list postings, however, came from a broader range of libraries. The overall group of respondents therefore has more diverse representation and includes public, private, university, government/national, and special libraries. The geographic distribution of the respondents includes libraries from the United States, Canada, and the United Kingdom. Fifteen libraries participate in the BIBCO program of the PCC. Twenty participants are OCLC member libraries, 2 are exclusively RLIN libraries, and 14 contribute catalog records to both utilities. One library catalogs solely in its own system.

Although the initial query was designed to identify willingness to participate in a survey and some responses indicated only yes or no to the three questions, 54 respondents supplied additional comments about the DCRB Core standard or characterized their cataloging practices or their collections. Of the 54 initial respondents, 24 did not wish to participate further, but included comments that will be discussed in a section in the following analysis. In addition, the six libraries that received the survey but did not return it provided some information about their reasons for using or not using the core standard. Their comments will also be included in the following discussion as indicators of perceptions about the DCRB Core standard.

\section{The Survey and Results}

The survey document (see appendix) contained 12 questions, most of which included multiple parts. The first two questions sought information about the type of library, the rare book collection, and the catalogers of the collection. Succeeding questions (3-5) asked about the original cataloging policies of the library, whether the DCRB Core standard has been considered, what decision has been made about its use, and whether any DCRB Core cataloging records have been created. Questions in the next part of the survey (6-9) suggested reasons for either using or not using the core standard. Further questions asked how DCRB Core records created by other institutions are handled (question 10) and whether any studies of users' perceptions have been done (question 11). Space for other comments was provided in question 12 .

While it was expected that the institutional data would vary from the responding libraries, questions 1 and 2 were asked to determine ranges of information to characterize the participants. No clear conclusions can be drawn about the relevance of the type of library, the size of the collection, the number of volumes cataloged or in the backlog, the number of rare book catalogers, or the reporting hierarchy within the institution. Both large and small collections are represented among the responses, ranging between a few hundred rare volumes in a collection (1 library) to close to or more than one million rare volumes (4 libraries), with dozens to more than 175,000 volumes in the backlog. The number of original rare book catalogers ranges from .25 full-time equivalents (FTE) to 9 FTE. Only 8 of the 37 libraries have more than 1 FTE rare book cataloger, 11 have less than one FTE, and 2 did not specify a number; the remaining 16 have only a single full-time cataloger. The questions about the size of the collection and the degree to which it is cataloged fully, minimally, or not at all seemed to pose more problems for the respondents than any other questions. Several respondents indicated they would be delayed in returning the questionnaire until they were able to determine the statistical information about their collections. Rare book catalogers report to cataloging or technical services departments in 19 of the responding libraries, to special collections departments in 12 libraries, and to both departments in 6 libraries.

The survey asked about the cataloging policies of the libraries to determine how many libraries use full-level DCRB or full-level AACR2 for cataloging pre-1801 books (questions 3.b.i and ii). The answers were not as straightforward as expected. In responding to how much original cataloging follows the full DCRB standard, 11 libraries responded that they do all their pre-1801 cataloging using that standard, and 13 libraries responded that they catalog none of their books using the standard. When answering the reverse question of how much original cataloging of pre1801 books follows the AACR2 full standard, 14 libraries indicated all and 15 libraries indicated none. One library catalogs none of its books according to either standard, but instead creates bibliographic records that are fuller than either DCRB or AACR2. Among libraries that do not adhere strictly to one standard, 12 libraries answered that they catalog some of their sixteenth- to eighteenth-century books using the full DCRB standard, and 7 indicated that they use full-level AACR2 for some of their books. The differences can only be attributed to the complexity with which catalogers of rare material view their work and to exceptions in cataloging practices.

To begin the inquiry about the use of DCRB Core by the responding libraries, four questions (3.b.iii-v and 4) were asked: have you considered using the DCRB Core standard, have you decided to use it, have you decided not to use it, and have you actually used it? Eighteen of the 37 libraries (48.6\%) have considered using the DCRB Core, 14 $(37.8 \%)$ have decided not to use it, and 6 (16.2\%) have decided they will use it. Six libraries have, in fact, created records using the standard. The 6 libraries that have decided to use DCRB Core are not the same 6 that actually have 
used the standard. One library said that the decision has been made to use the standard, but implementation has been hindered by staffing difficulties. Another library has applied the core standard without having made the policy decision that it expects to make in the near future. Two other libraries expressed their intention to use the standard in the future, one as soon as appropriate collections have been identified. Depending on the answer to the question of use of the DCRB Core, respondents were asked to indicate why and how they use the standard (questions 5-8) or why they do not (question 9). In each case, a list of reasons was given for potential responses with additional space for other comments.

In the discussion that follows, the responses of those libraries that use the DCRB Core standard will precede the responses of those libraries that do not use the standard. Each of the 6 libraries that use the DCRB Core did not answer all the questions; and since the number is small, only the positive responses will be noted. One library uses the DCRB Core standard to catalog all of its sixteenth- to eighteenth-century books. Four use DCRB Core for some of their cataloging; 1 among them uses the standard as the default, but enhances the record if there is a compelling reason. The sixth library uses the DCRB Core to catalog its rare books even though none are pre-1801 imprints. Three of the 6 libraries use the DCRB Core for particular reasons: for a specific collection or when lack of expertise or want of a significant reference work would make it impossible to catalog at the full level. All 6 have applied the standard at cataloger discretion. and 2 of the 6 also have applied it at curator discretion.

In response to why and how they use the DCRB Core standard, four libraries answered that they use it to save time, three to increase production, four to gain faster control over their backlog, and three because it is more costeffective. In addition, one librarian whose institution does not hold any pre-1801 imprints replied that they use the DCRB Core because it is "better to capture the uniqueness of what we own in the archival and special collections department," and another respondent noted that they upgrade to the level of the DCRB Core standard some of the brief records for early imprints that can be found in the OCLC database. Only 1 library uses the standard as it is written, "since it is written to be flexible"; 2 other libraries sometimes use it as written. Four libraries add more fields than the standard requires. The additional fields include: notes for contents (505), immediate source of acquisition (541), and ownership and custodial history (561); genre, form, or physical characteristic terms (655); added titles (246 and 740); and local information required by the library's online system or cataloging policies. Five of the libraries encourage cataloger's discretion to determine whether to include additional fields and what the fields should be. The 6 libraries indicated the percentage of bibliographic records created using the DCRB Core standard to be less than $1 \%, 1 \%$, less than $5 \%$, less than $10 \%$, and $100 \%$, with one library reporting that no statistical records had been kept for DCRB Core records. In actual numbers 4 libraries reported they have created the following numbers of records: $10,10-15,18$, and ca. 4,400. The fifth and sixth libraries were unable to give figures.

The library that catalogs all its sixteenth- to eighteenthcentury books using the DCRB Core standard does not add bibliographic records immediately to OCLC, and there may be a period of time before they appear in the utility's database. The library that said it starts with the DCRB Core record as the default and enhances as necessary, creates its original catalog records locally without an 040 field (that would contain $\$$ e derb) because the generic 040 with the library's holding symbol (but without $\$ \mathrm{e}$ dcrb) is added automatically when the records are sent to OCLC. This library has not kept records of the number of DCRB Core records created. Given that these 2 libraries may create a considerable number of DCRB Core records without a way to track them in OCLC and that other libraries, that they have created a minimal number of DCRB Core records, it would be surprising if the national databases have a significant number of DCRB Core records.

The initial responses of the 2 libraries that said they have used the DCRB Core but did not return the survey indicate that they probably have created few catalog records using the standard. When 1 of the 2 libraries applies the DCRB Core, it does so only for seventeenth- and eighteenth-century books, not for fifteenth- and sixteenth-century books, and it never omits notes justifying added entries. The other library applies the standard in limited instances because it feels that rare books should be given full-level cataloging. Since the DCRB Core does not require a standard call number, this second library can contribute BIBCO records for broadsides and pamphlets that it does not classify. Adding these 2 libraries that did not participate in the survey to the 6 that did participate brings the total of known users of the DCRB Core standard to 8 .

The 31 libraries that do not use the DCRB Core standard indicated their reasons in response to a ten-part question that suggested 9 possible reasons and asked for others in the tenth part (question 9). As indicated in table 1, each of the reasons was affirmed by some of the libraries. That the DCRB Core standard is inferior was the reason cited by most of the libraries, 19 (61.3\%), that have not used it in their original cataloging records for rare books. Fifteen respondents $(48.4 \%)$ said that the description is not accurate enough and that there are not enough access points with the use of the standard. Eleven $(35.5 \%)$ stated that they have not used the DCRB Core because it would require learning a new standard, and $8(25.8 \%)$ said they do not use the stan- 
dard because they are unfamiliar with it. Six respondents (19.4\%) reported shortages or changes in staff that have kept them from using the standard. Five libraries (16.1\%) said that training is unavailable, and 5 also indicated that the use of the DCRB Core standard would disrupt the established workflow. Only 4 (12.9\%) said they do not have material appropriate to catalog using the standard.

The opinion and perception of nearly two-thirds of the libraries responding that they do not use the DCRB Core standard is that the DCRB Core record is inferior to the full record. Almost half think that the standard does not provide enough access points and that the description is not accurate enough. Clearly these three most prevalent perceptions indicate that the survey respondents have not been willing to give up fuller bibliographic treatment for their rare books. This opinion of the lesser quality of the DCRB Core record as a reason for nonadoption by most of the respondents is reminiscent of a similar kind of negative perception and resistance to acceptance that were noted for the Books Core by Cromwell $(1994,423-24)$ in her observation that cataloger's attitude and acceptance are needed for the success of the Books Core. The six other reasons that the respondents affirmed for not using the DCRB Core standard are all operational obstacles within their libraries more than opinions or perceptions. Changes in libraries' current personnel, procedures, or materials issues might create more acceptable conditions for the adoption of the DCRB Core standard. Catalogers could learn the new standard or become familiar with it, changes or shortages in staff could be overcome with time or more money, a new workflow could be established, training could be sought, and appropriate materials might be acquired.

The participants gave a number of other reasons, however, for not undertaking use of the DCRB Core. In response to the possibility that a library may not have appropriate materials for using the core standard, some libraries qualified that reason noting the small quantity of rare books they catalog. Seven libraries said that they do not catalog many rare books, that they do so little original cataloging, especially of rare books, or that they have so few pre-1801 imprints that it is easier to apply the full standard and not worth applying a different standard when they encounter a title that may be eligible for core-level cataloging. One librarian replied that the categories of materials that would be candidates for DCRB Core-level cataloging are generally cataloged to their own minimal-level standard. Administrative or departmental policy to catalog all books at full-level DCRB was cited by 6 libraries as the reason for not applying the DCRB Core standard. Although 13 libraries answered that they do not use full-level DCRB for cataloging any of their sixteenth- to eighteenth-century books, 4 specifically reiterated that they use only AACR2 full for cataloging rare books. Two libraries emphasized their production goals and decisions to apply
Table 1. Thirty-one Survey Respondents' Ranked Choices for Not Using the DCRB Core Standard (from Question 9)

\begin{tabular}{lrrrc}
\hline Reasons & Yes & \% Yes & No & Unanswered \\
Core is inferior & 19 & 61.3 & 11 & 1 \\
Description not accurate enough & 15 & 48.4 & 14 & 2 \\
Too few access points & 15 & 48.4 & 16 & - \\
Requires learning new standard & 11 & 35.5 & 18 & 2 \\
Unfamiliarity with standard & 8 & 25.8 & 22 & 1 \\
Shortages or changes in staff & 6 & 19.4 & 24 & 1 \\
Use would disrupt workflow & 5 & 16.1 & 24 & 2 \\
Training is unavailable & 5 & 16.1 & 25 & 1 \\
Material not appropriate & 4 & 12.9 & 27 & - \\
Additional reasons & 18 & 58.1 & - & 13 \\
\hline
\end{tabular}

certain standards as reasons for not taking time to learn a new standard. One librarian thinks "the introduction of a DCRB Core standard runs contrary to the whole intent of DCRB to provide fuller description than AACR2." Another librarian said that not justifying access points is confusing to users and that if the cataloger starts to add more fields, it would be easier to create full-level records. Two respondents said their reference collections are not comprehensive enough to provide references in a 510 field to allow the abbreviated description of the DCRB Core standard. Two other libraries indicated that their backlogs are not large enough to need to implement DCRB Core cataloging. Four librarians each offered one of the following reasons: use of the Core does not increase production, it would create more inconsistencies in the catalog than are already there from so many changes in standards over the years, there is little time for the catalogers to assimilate the new standard, and catalogers prefer one standard not choices. These additional reasons and comments express strong opposition to the use of the DCRB Core standard. For the most part, they are internal or operational issues that pose obstacles to the adoption of the standard.

Remarks from the 24 libraries that did not wish to participate in the survey provide further insight into why some libraries do not use the DCRB Core. Their reasons are categorized in table 2. Some of the reasons are identical to those of the survey participants: the rare book collection is not sufficiently large to warrant learning to catalog by any standard other than full-level DCRB (11 libraries); the library is not familiar with or even aware of DCRB Core (6 libraries); there are changes (5 libraries) or shortages (4 libraries) in staff; the DCRB full standard is not used (4 libraries); the policy is to catalog at the full level (3 libraries); and training poses a problem (1 library). In addition to these commonly held reasons for not applying the DCRB Core, 3 libraries indicated that they do not use any of the core standards, and 2 libraries hesitate to use the DCRB Core because it might prohibit a non-BIBCO library from enhancing the core record to a full-level record in OCLC. 
Table 2. Categorized Reasons for Not Using DCRB Core from Twenty-four Nonparticipants in Survey

\begin{tabular}{lr}
\hline Reasons & No. \\
Not enough of a collection to make it worthwhile & 11 \\
Not informed enough or aware of DCRB Core & 6 \\
Cataloger is retiring or have a change of staff & 5 \\
Do not use DCRB full & 4 \\
Have a shortage of staff & 4 \\
Do not use any core standard & 3 \\
Policy is to catalog at full level (including BIBCO records) & 3 \\
Other libraries would not be able to enhance records to full level & 2 \\
Feels the need to justify added entries & 1 \\
Feels limitation in training & 1 \\
Feels limitation in knowledge & 1 \\
Does not do BIBCO for books & 1 \\
Has not cataloged rare books in a while & 1 \\
Does not create BIBCO records for rare books & 1 \\
Only a few catalogers are trained in DCRB & 1 \\
Too little time to discuss issues related to DCRB Core & 1 \\
Has a large backlog & 1 \\
Uses Books Core & 1 \\
Catalogs few original records for rare books & 1 \\
Differences between core and full are minor & 1 \\
Using core does not save much time & 1 \\
Doubts can convince Special Collections of value of core records & 1 \\
E-resources are becoming priorities & 1 \\
\hline
\end{tabular}

Each of the following reasons was cited by a single library for not employing the DCRB Core standard: added entries should be justified in a note; knowledge is too limited to apply the standard; BIBCO books records are not created; BIBCO rare books records are not created; rare books have not been cataloged recently; only a few catalogers are trained to use DCRB; too little time is available to discuss DCRB Core and issues related to it; the backlog is large; the Books Core is used instead; too few original records for rare books are created; the differences between core and full level are minor; the core standard does not save much time; the value of DCRB Core records cannot be sold to Special Collections; and, finally, e-resources are becoming more of a cataloging priority than printed special collections materials. Common emphases and implications that run through many of these reasons are the little availability of time and the desire for adherence to fuller standards, two characteristics that have long been in competition in technical services processes in libraries. One library, however, intends to use the DCRB Core standard, and two others are considering using it.

How well cataloging copy is viewed and used by other libraries is one way of evaluating the efficacy of a cataloging standard. Responding to question 10, 9 participants in the survey said their copy catalogers accept DCRB Core records without changing them, although 1 library said there was room for cataloger's judgment and 2 libraries indicated that they might add subject headings or local notes. Nineteen respondents said their copy catalogers edit DCRB Core records locally. In response to the question of whether original catalogers enhance DCRB Core records to full level locally, 29 libraries answered in the affirmative; 5 do not enhance core records to full level locally. At 16 libraries, original catalogers enhance DCRB Core records to full level nationally; at 16 libraries they do not enhance nationally, although 2 libraries said they may do so in the future. Four of the respondents noted that their catalogers have never seen a DCRB Core record; 1 of them would edit if a core record were encountered. Since there is so little evidence for the creation of DCRB Core records, it is surprising that not more libraries commented that they have not seen them in the bibliographic utilities. Further research in the databases of the bibliographic utilities is needed to confirm that the DCRB Core standard has as yet found little acceptance and use among rare book catalogers and curators.

The final question on the survey (question 11) asked if any use studies of DCRB Core records have been conducted. Not surprisingly, none of the respondents has done such a survey. In their additional comments, however, three librarians said they think the purported time savings of core cataloging records are not worth the cost to the users in locating materials they need. These opinions are greatly supported by many special collections departments' preferences for using the DCRB full standard.

\section{Conclusions}

Clearly, the preponderance of the evidence indicates that most libraries surveyed have not used the DCRB Core standard and prefer to catalog their rare books using a full standard, whether DCRB or AACR2. The DCRB Core standard was not written as a replacement for full-level cataloging for all rare books. The standard states that the expectation is that materials cataloged using the DCRB Core will be chosen more for their accessibility than for full bibliographic treatment (PCC 1999a). Eight libraries (six survey participants and two that did not participate) have identified appropriate collections to catalog using the DCRB Core standard. Among the responses received both in the initial query and in the survey itself, six additional libraries indicated that although they do not now use the DCRB Core standard in their cataloging, they are expecting to do so in the future or seriously want to consider using it. The total of fourteen libraries that either do use the DCRB Core or almost assuredly will use it in the future indicates the beginning of acknowledgement of the value of the standard and a more positive attitude among some catalogers toward the standard.

A recent survey of the special collections departments of the ARL libraries finds that large portions of the collections are uncataloged (Panitch 2001, 49). Although formats other 
than books form the bulk of the uncataloged collections, an average of $15 \%$ of the book collections have no access through any form of bibliographic description, and $49 \%$ have access only through a card catalog. Many non-ARL libraries may also have large proportions of their special collections in uncataloged backlogs. According to the minutes of recent meetings of the ARL Task Force on Special Collections, one primary issue under discussion is the question of providing increased access to those backlogged materials through bibliographic control (ARL Task Force on Special Collections 2002).

Many of the books in backlogs may have existing bibliographic records in OCLC and RLIN that can be used by copy catalogers. For those books that do not already have catalog records in the bibliographic utilities, however, the application of the DCRB Core standard in creating original bibliographic records has potential. To begin a project to catalog backlogged material using the DCRB Core standard, a primary task for special collections librarians is to identify specific collections that will be appropriate for DCRB Corelevel cataloging. One library among the survey respondents is considering cataloging its early French pamphlets using the DCRB Core. Other pamphlet collections or subject collections, such as political or religious tracts, may be candidates for DCRB Core cataloging. One librarian said that using a cataloging template for materials issued by the same publisher would make DCRB Core cataloging even more efficient. Several libraries indicated that they are looking for appropriate collections. One respondent noted that sometimes the DCRB Core standard is applied to enhance a brief cataloging record to bring it up to a higher level without the necessity of enhancing to the full level.

In addition to identifying collections that are candidates for DCRB Core cataloging, libraries will need to encourage cataloger's judgment to help overcome the resistance to using core standards. Because the flexibility of the core standard permits a wide range of additional elements in the core record, the cataloger is not limited to the bare minimum requirements. Not every book in a backlog will be a candidate for core-level cataloging, but identifying the proper collections and undertaking DCRB Core-level cataloging projects can help increase accessibility to those materials that users do not know exist in rare book collections.

\section{Works Cited}

Association of College and Research Libraries. Rare Books and Manuscripts Section. Bibliographic Standards Committee (ACRL RBMS BSC). 1998a. Bibliographic Standards Committee meeting minutes, midwinter and annual. Accessed Dec. 7, 2001, www.folger.edu/bsc/1998midwin.html and www.folger.edu/bsc/1998annual.html.

- 1998b. List of optional rules in DCRB. Accessed Dec. 14, 2001, www.lib.byu.edu/ catalog/people/rlm/bsc/coreopt. $\mathrm{htm}$. Also available at www.folger.edu/bsc/coreopt.html.
Association of Research Libraries (ARL). Task Force on Special Collections. 2002. ARL Special Collections Task Force minutes, April 9, 2002, and June 15, 2002. Accessed July 26, 2002, www.arl.org/collect/spcoll/tforce/min0402.html and www.arl. org/collect/spcoll/tforce/min0602.html.

Banush, David. 2001. BIBCO core record study: Final report: Prepared for the PCC Policy Committee. Accessed Sept. 24, 2001, www.loc.gov/catdir/pcc/bibco/coretudefinal.html.

Cromwell, Willy. 1994. The core record: A new bibliographic standard. Library Resources and Technical Services 38: 415-24.

Czeck, Rita L. H., Elizabeth Icenhower, and Charlene Kellsey. 2000. PCC core records versus PCC full records: Differences in access? Cataloging and Classification Quarterly 29, no. 3: 81-92.

Exlibris. 1997-1998. Exlibris: List archives. Accessed Oct. 2, 2001, http://palimpsest.stanford.edu/byform/mailing-lists/exlibris/.

Fletcher, Jain. 1999. DCRB Core: Examples [prepared for the Association of College and Research Libraries, Rare Books and Manuscripts Section, Bibliographic Standards Committee]. Accessed Oct. 25, 2001, www.lib.byu.edu/ catalog/people/ $\mathrm{rlm} / \mathrm{bsc} /$ corexx.htm.

Hyslop, Colleen F. 1997. Highlights of the Program for Cooperative Cataloging: The core record and consolidation of CONSER and PCC. From Catalog to Gateway 11. Accessed Aug. 2, 2002, www.loc.gov/catdir/pcc/hyslop2.html. Originally published as a supplement to ALCTS Newsletter 8, no. 4, 1997.

Kelley, Sherry L., and Brian E. C. Schottlaender. 1996. UCLA/OCLC Core Record Pilot Project: Preliminary report. Library Resources and Technical Services 40, no. 3: 251-60.

Lange, Holley R. 1998. Creating core records for federal documents: Does it make a difference? Cataloging \& Classification Quarterly 26, no. 3: 87-94.

Letarte, Karen M., and Michelle R. Turvey. 2001. User perspectives on the Program for Cooperative Cataloging BIBCO core record standard: A pilot study of the core bibliographic record for books: Final report. Accessed Oct. 30, 2001, www.loc.gov/catdir/pcc/modelcfinal.pdf.

Panitch, Judith M. 2001. Special collections in ARL libraries: Results of the 1998 survey sponsored by the ARL Research Collections Committee. Washington, D.C.: Association of Research Libraries.

Program for Cooperative Cataloging (PCC). 1996. Core bibliographic record for books. Accessed Sept. 24, 2001, www.loc.gov/catdir/pce/corebook.html.

. 1999a. Core standard for rare books (DCRB Core). Accessed Sept. 24, 2001, www.loc.gov/catdir/pce/dcrbcore. html.

. 1999b. Introduction to the Program for Cooperative Cataloging BIBCO core record standard. Accessed Sept. 24, 2001, www.loc.gov/catdir/pcc/bibco/coreintro.html.

. Task Group for Developing a Standard for Core Treatment of Rare Books (PCC Task Group). 1998. DCRB Core Task Group: Final report. Accessed Sept. 24, 2001, www.loc.gov/catdir/pcc/dcrbreport.html.

Thomas, Sarah E. 1996. The core bibliographic record and the Program for Cooperative Cataloging. Cataloging and Classification Quarterly 21, no. 3/4: 91-108. 


\section{Appendix Survey Questions}

1. Information about your institution:

a. Is your institution public or private?

b. Is your library a public, college, university, special, government, or national library?

c. Does your library contribute original bibliographic records to OCLC or to RLIN?

2. Information about your rare book collection and catalogers:

a. How many volumes do you have in your rare book collection?

b. How many volumes are cataloged fully?

c. How many volumes are cataloged minimally?

d. How many volumes are in your backlog?

e. How many original catalogers (FTE) are on your rare book cataloging team?

f. How many copy catalogers (FTE) are on your rare book cataloging team?

g. Do your rare book catalogers report to the Cataloging Department, the Special Collections Department, the Cataloging Department within Special Collections, or another unit?

3. Information about your original cataloging policy:

a. For nonrare books:

i. Is it your library's policy to use or not to use the PCC core standards for books and other formats?

b. For rare books:

i. Is it your policy to catalog all, some, or none of your sixteenth- to eighteenth-century books using the DCRB full standard?

ii. Is it your policy to catalog all, some, or none of your sixteenth- to eighteenth-century books using the AACR2 full standard for books?

iii. Have you considered using the DCRB Core standard for cataloging your sixteenth- to eighteenth-century books?

iv. Have you made a decision to use the DCRB Core standard?

v. Have you made a decision not to use the DCRB Core standard?

4. Have you used the DCRB Core record for cataloging rare books? (If no, go to question 9.)

5. What determines your application of the DCRB Core standard? (Please answer all that apply.)

a. Do you catalog all, some, or none of your sixteenth- to eighteenth-century books using the DCRB Core standard?

b. If you catalog only some rare books using the core standard, do you use it to catalog particular categories of materials, e.g., those selected by date, subject, or collection?

c. If yes, what categories of materials?

d. Is the standard applied at cataloger discretion?

e. Is it applied at curator discretion?

6. Why do you use the DCRB Core standard? (Please answer all.)
a. Do you use it to save time in cataloging?
b. Do you use it to increase production?
c. Do you use it to help gain faster bibliographic control over a backlog?
d. Do you use it because it is more cost-effective?
e. Do you use it for any other reason? (Please specify.)

7. Frequency of use of DCRB Core standard:

a. What percentage of your cataloging records is created with the DCRB Core standard?

b. Do you use it as often as your library uses the core record for books and other formats?

c. Approximately how many DCRB Core records have you created since the approval of the standard in January 1999?

8. How do you use the DCRB Core standard?

a. Do you use the DCRB Core standard as it is written?

b. Or have you decided routinely to include more fields than the standard defines, e.g., more 500 fields? 
c. What additional fields have you decided to include routinely?

d. Do you encourage cataloger discretion in determining what additional fields to include?

9. If you do not use the DCRB Core record, what are the reasons? (Please answer all.)

a. That you do not have material appropriate to catalog using the core standard?

b. That it does not describe a book accurately enough for your users to identify what they need?

c. That it does not give enough points of access to the material?

d. That the core record is inferior to the full record?

e. That its use would create a disruption of the established workflow?

f. That its use would require catalogers to learn or accommodate a standard different from the one in long-time practice

g. That training is not available?

h. That you are not familiar enough with the standard?

i. That shortages or changes in staff do not make it practical to learn a different standard?

j. Any other reason? (Please specify.)

10. Your use of the DCRB Core record created by other libraries:

a. If you have copy catalogers working on rare books, do they accept DCRB Core records as they encounter them, or do they edit them locally (other than adding call numbers)?

b. Do your catalogers (copy or original) ever enhance DCRB Core records to full level locally?

c. Do they ever enhance DCRB Core records to full level nationally in one of the bibliographic utilities?

11. Studies to evaluate the DCRB Core from the patron perspective:

a. Have you undertaken any studies to evaluate the DCRB Core standard from the patron perspective?

b. If so, what evaluation criteria did you use?

c. Would you be willing to share your evaluation criteria?

12. Do you have any other comments about the DCRB Core standard? 